\title{
IDENTIFYING RESISTANCE TO ROOT-KNOT NEMATODES IN Capsicum GENOTYPES
}

\author{
IDENTIFICAÇÃO DE GENÓTIPOS DE Capsicum RESISTENTES A NEMATOIDES \\ DE GALHA
}

\author{
Renato Silva SOARES ${ }^{1}$; Edgard Henrique Costa SILVA ${ }^{1}$; Willame dos Santos CANDIDO'; \\ Guilherme Matos Martins DINIZ ${ }^{1}$; Francisco José Becker REIFSCHNEIDER ${ }^{2}$; \\ Pedro Luiz Martins SOARES ${ }^{1}$; Leila Trevisan BRAZ ${ }^{1}$ \\ 1. Department of Plant Production, UNESP, Jaboticabal, SP, Brazil; 2. Embrapa International Relations, Brasília, DF, Brazil. \\ renato_2366@hotmail.com.
}

\begin{abstract}
The present study aimed to evaluate Capsicum accessions for resistance to Meloidogyne incognita race 3, Meloidogyne javanica and Meloidogyne enterolobii. Two experiments with different genotypes of hot and sweet peppers were carried out in a completely randomized design. The first experiment was conducted in a $31 \times 3$ factorial scheme with 27 genotypes of Capsicum annuum, two cultivars of hot pepper, one line of Capsicum frutescens and tomato 'Santa Cruz Kada', and three species of nematodes (M. incognita race 3, M. javanica and M. enterolobii). In the second experiment, we used a factorial scheme $39 \times 3$ with 36 accessions of $C$. annuum, two hot pepper cultivars and the 'Santa Cruz Kada' tomato and three nematodes species mentioned earlier. The total number of eggs and second-stage juveniles (TNEJ), number of eggs and second-stage juveniles per gram of root (NEJGR), reproduction index (RI) and reproduction factor (RF) were evaluated. Based on RI and RF, the genotypes CNPH 185, CNPH 187 and CNPH 680 were resistant and very resistant to $M$. incognita race 3 and $M$. javanica, simultaneously. The $C$. frutescens line presented resistance to the three root-knot nematode species.
\end{abstract}

KEYWORDS: Meloidogyne incognita. Meloidogyne javanica. Meloidogyne enterolobii. chili and sweet peppers. reaction.

\section{INTRODUCTION}

Cropping of sweet and hot peppers (Capsicum spp.) is gaining notoriety in the Brazilian market, due to the growing demand in the segment of fresh vegetables, condiments, seasonings and preserves. Among the domesticated species of Capsicum, the sweet pepper (Capsicum annuum L.) stands out for the high yield and economic value (BÜTTOW et al., 2010; PIMENTA et al., 2016), with an estimated national production area of 12.000 hectares (MOURA et al., 2012). In Brazil, the main producing regions are the Southeast and CenterWest, especially the state of São Paulo, which produced, in the 2012 harvest, 65.6 thousand tons in 2.2 thousand hectares (IEA, 2016).

With the increase of sweet pepper consumption, cropping mainly carried out in protected environment, due to the greater productivity and fruit quality, as well as the regularization of product supply throughout the year (PINHEIRO et al., 2014). However, successive crops, together with inadequate soil and crop management, have led to a rise in root diseases, especially root-knot nematodes.
The Meloidogyne spp. nematodes are phytoparasites that have caused serious damages in cropping of hot and sweet peppers. Several studies have reported the parasitic action of these nematodes on Capsicum (MELO et al., 2011; GONÇALVES et al., 2014; PINHEIRO et al., 2014; PINHEIRO et al., 2015).

Genetic control is the most sustainable way to manage root-knot nematodes, since it poses no risk to human health; it is relatively of low cost and does not pollute the environment (HUSSAIN; MUKHTAR; KAYANI, 2014; LIU et al., 2015). Lópes-Pérez et al. (2006) point out that the use of genetic resistance is an attractive alternative because it does not require major adaptations in the productive procedures of the property.

It is known that 90 species are described belonging to the genus Meloidogyne (MOENS et al., 2009). Among them, Meloidogyne incognita (Kofoid \& White) and Meloidogyne javanica (Treub) are the most important species for the sweet pepper (PINHEIRO et al., 2014). Recently, the species Meloidogyne enterolobii Yang and Eisenback (sin. Meloidogyne mayaguensis Rammah and Hirschmann) has gained importance, as effective sources of resistance against the major 
species of Meloidogyne have been shown to be ineffective in its control (BRITO et al., 2007; PINHEIRO et al., 2013).

To minimize losses caused by nematodes occurrence, croppers have adopted the grafting technique, using resistant rootstocks. There are some commercial hybrids available for sweet peppers rootstocks, such as 'Silver' and 'Snooker', both resistant to $M$. incognita and $M$. javanica (PINHEIRO et al., 2014). However, these rootstocks do not present resistance to $M$. enterolobii.

There are no reports of sweet pepper cultivars with multiple resistance to root-knot nematodes, being the search for Capsicum genotypes that, simultaneously, present resistance to the major Meloidogyne species, is of fundamental importance for the development of resistant cultivars or rootstocks. Thus, this study aimed to assess Capsicum genotypes for resistance to $M$. incognita race $3, M$. javanica and $M$. enterolobii.

\section{MATERIAL E METHODS}

For the identification of resistant genotypes to the species of root-knot nematodes, two experiments were carried out consecutively in a greenhouse in the Sector of Vegetable Crops and Aromatic Medicinal Plants and Plant Pathology Laboratory, Department of Plant Protection, Universidade Estadual Paulista (UNESP), Faculdade de Ciências Agrárias e Veterinárias (FCAV), Jaboticabal (2115'22" S, 48¹8'58" W; $595 \mathrm{~m}$ a.s.1.), São Paulo, Brazil, between the months of September of 2015 to February of 2016.

A total of 63 genotypes of Capsicum annuum, two commercial pepper cultivars (BRS Moema and BRS Mari), one sweet pepper cultivar (Ikeda) and one chilli pepper strain (C. frutescens) were evaluated for resistance to $M$. incognita race 3 , M. javanica and M. enterolobii.

The accessions of Capsicum annuum are part of the collection of peppers and sweet peppers present from the Active Germplasm Bank of Embrapa Hortaliças, these being from collections and/or partnerships with national and international institutions. Table 1 shows the relation of the genotypes used, as well as the origin and main morphological characteristics.

Table 1. Origin and main characteristics of 63 genotypes of Capsicum annuum from the Active Germplasm Bank of Embrapa Hortaliças, evaluated in two experiments on the reaction to root-knot nematodes.

\begin{tabular}{|c|c|c|c|c|}
\hline $\mathbf{N}^{\mathbf{0}}$ & Genotypes & Origin & Fruit color & Fruit format \\
\hline & Experiment & & & \\
\hline 1 & CNPH 29 & FAO* & Dark red & Elongated \\
\hline 2 & CNPH 30 & Spain & Dark red & Rectangular \\
\hline 3 & CNPH 31 & FAO & Dark red & Elongated \\
\hline 4 & CNPH 32 & Japan & Dark red & Rectangular \\
\hline 5 & CNPH 33 & USA & Red & Triangular \\
\hline 6 & CNPH 40 & Japan & Red & Elongated \\
\hline 7 & CNPH 42 & Japan & Dark red & Elongated \\
\hline 8 & CNPH 43 & Japan & Dark red & Elongated \\
\hline 9 & CNPH 44 & Japan & Red & Elongated \\
\hline 10 & CNPH 45 & Japan & Red & Elongated \\
\hline 11 & CNPH 47 & USA & Dark red & Triangular \\
\hline 12 & CNPH 48 & USA & Red & Elongated \\
\hline 13 & CNPH 66 & Brazil & Red & Triangular \\
\hline 14 & CNPH 67 & Brazil & Dark red & Rectangular \\
\hline 15 & CNPH 68 & Brazil & Dark red & Triangular \\
\hline 16 & CNPH 69 & Brazil & Dark red & Triangular \\
\hline 17 & CNPH 144 & Malaysia & Dark red & Elongated \\
\hline 18 & CNPH 147 & France & Dark red & Rectangular \\
\hline 19 & CNPH 149 & Mexico & Red & Triangular \\
\hline 20 & CNPH 150 & Argentina & Red & Rectangular \\
\hline 21 & CNPH 183 & Guatemala & Yellow/Orange & Rectangular \\
\hline 22 & CNPH 184 & India & Dark red & Rectangular \\
\hline
\end{tabular}




\begin{tabular}{|c|c|c|c|c|}
\hline 23 & CNPH 185 & Mexico & Dark red & Elongated \\
\hline 24 & CNPH 186 & Mexico & Dark red & Triangular \\
\hline 25 & CNPH 187 & Mexico & Dark red & Triangular \\
\hline 26 & CNPH 188 & India & Dark red & Rectangular \\
\hline 27 & CNPH 190 & India & Dark red & Rectangular \\
\hline \multicolumn{5}{|c|}{ Experiment 2} \\
\hline 28 & CNPH 64 & USA & Red & Elongated \\
\hline 29 & CNPH 145 & Malaysia & Dark red & Elongated \\
\hline 30 & CNPH 191 & Brazil & Red & Triangular \\
\hline 31 & CNPH 194 & Spain & Red & Triangular \\
\hline 32 & CNPH 198 & Argentina & Dark red & Rectangular \\
\hline 33 & CNPH 199 & Argentina & Red & Rectangular \\
\hline 34 & CNPH 200 & Argentina & Red & Rectangular \\
\hline 35 & CNPH 291 & USA & Yellow/Orange & Rectangular \\
\hline 36 & CNPH 292 & USA & Dark red & Rectangular \\
\hline 37 & CNPH 295 & USA & Red & Rectangular \\
\hline 38 & CNPH 296 & USA & Red & Rectangular \\
\hline 39 & CNPH 297 & USA & Red & Rectangular \\
\hline 40 & CNPH 432 & Figi & Dark red & Elongated \\
\hline 41 & CNPH 433 & Taiwan & Red & Rectangular \\
\hline 42 & CNPH 580 & Netherlands & Red & Rectangular \\
\hline 43 & CNPH 581 & Netherlands & Red & Rectangular \\
\hline 44 & CNPH 582 & Netherlands & Red & Rectangular \\
\hline 45 & CNPH 583 & Netherlands & Red & Rectangular \\
\hline 46 & CNPH 593 & Netherlands & Red & Rectangular \\
\hline 47 & CNPH 602 & USA & Dark red & Triangular \\
\hline 48 & CNPH 640 & Hungary & Red & Triangular \\
\hline 49 & CNPH 641 & Hungary & Dark red & Pitanga type \\
\hline 50 & CNPH 642 & Hungary & Red & Triangular \\
\hline 51 & CNPH 644 & Hungary & Dark red & Rounded \\
\hline 52 & CNPH 646 & Hungary & Dark red & Elongated \\
\hline 53 & CNPH 677 & Iran & Dark red & Triangular \\
\hline 54 & CNPH 680 & USA & Dark red & Triangular \\
\hline 55 & CNPH 682 & India & Dark red & Elongated \\
\hline 56 & CNPH 683 & India & Dark red & Triangular \\
\hline 57 & CNPH 684 & Spain & Red & Triangular \\
\hline 58 & CNPH 687 & Turkey & Dark red & Triangular \\
\hline 59 & CNPH 688 & Turkey & Red & Triangular \\
\hline 60 & CNPH 690 & Turkey & Dark red & Triangular \\
\hline 61 & CNPH 691 & Turkey & Red & Elongated \\
\hline 62 & CNPH 692 & Turkey & Dark red & Triangular \\
\hline 63 & CNPH 693 & Turkey & Dark red & Triangular \\
\hline
\end{tabular}

*FAO: Food and Agriculture Organization of the United Nations

Both experiments were conducted in a completely randomized design. The first experiment was arranged in a factorial scheme $31 \times 3$, being 27 genotypes of $C$. annuum (Table 1), the hot pepper cultivars BRS Moema and BRS Mari, a line of tabasco pepper ( $C$. frutescens) and the tomato 'Santa
Cruz Kada' used as a susceptibility control to the genus Meloidogyne spp., and three species of rootknot nematodes ( $M$. incognita race 3, M. javanica and $M$. enterolobii).

The second experiment was conducted in a factorial scheme $39 \times 3$, with 31 genotypes of $C$. 
annuum (Table 1), two hot peppers cultivars (BRS Moema and BRS Mari), the 'Santa Cruz Kada' tomato and three species of root-knot nematodes $(M$. incognita race 3, $M$. javanica and $M$. enterolobii). Both experiments contained six replicates and the plots were composed of one plant.

The subpopulations of $M$. incognita race 3, $M$. javanica and $M$. enterolobii were obtained from 'Santa Cruz Kada' tomato roots, belonging to the nematode collection of the Laboratory of Nematology, Department of Plant Protection, Faculdade de Ciências Agrárias e Veterinárias (UNESP), Campus of Jaboticabal.

We prepared the inoculum as described by Hussey and Barker (1973). The estimation of eggs and second-stage juveniles' population in the suspension was performed using a Peter's counting chamber under a photonic microscope, with a subsequent concentration adjustment for 1.000 eggs and second-stage juveniles $\mathrm{mL}^{-1}$.

The seedlings, in both experiments, were produced in 128 cells expanded polystyrene trays filled with Bioplant ${ }^{\circledR}$. Two seeds were disposed per cell, with subsequent thinning to obtain one quality seedling. We transplanted the seedlings at 40 days after sowing to $2.0 \mathrm{~L}$ plastic pots, containing the mixture of soil, sand and bovine manure, in the ratio $1: 1: 1$, previously autoclaved $\left(120^{\circ} \mathrm{C}, 1 \mathrm{~atm}, 1\right.$ hour $)$. At the time of transplantation, with the aid of an automatic pipette, we inoculated $5 \mathrm{~mL}$ of a suspension containing 5,000 eggs and second-stage juveniles $\left(J_{2}\right)$ in each pot, for each root-nematode species separately, characterizing the initial population (Pi).

After 90 days of inoculation, the plants were evaluated by separating between shoot and roots. We extracted eggs and other nematode stages according to the technique of Hussey and Barker (1973). With the aid of a Peter's chamber and a photonic microscope, we quantified the total number of eggs and second-stage juveniles (TNEJ). From this procedure, we obtained the final nematodes population (FP) in the roots.

To assess the resistance of genotypes to $M$. incognita race 3, M. javanica and M. enterolobii, we used the total number of eggs and second-stage juveniles (TNEJ), number of eggs and second-stage juveniles per gram of root (NEJGR), reproduction index (RI) and reproduction factor (RF). The number of eggs and $\mathrm{J}_{2}$ per gram of root was determined from the division of the TNEJ /root total weight. The reproduction factor was calculates as the following formula:

$$
\mathbf{R F}=\frac{f \mathrm{P}}{i \mathrm{P}}
$$

Where: $\mathrm{RF}=$ Reproduction factor, $f \mathrm{P}=$ Final population and $i \mathrm{P}=$ Initial population of viable eggs and second-stage juveniles. Plants with $\mathrm{RF}<1$ were considered resistant to the nematode, and with $\mathrm{RF} \geq 1$ were considered susceptible to the nematode, according to Oostenbrink (1966).

We calculated the reproduction index (RI) considering the 'Santa Cruz Kada' tomato as a susceptibility control $(100 \%)$ in relation to nematodes reproduction obtained in Capsicum genotypes. Thus, the formula was used: $100 \mathrm{x}$ (Number of eggs per gram of root of each replicate/Average number of eggs per gram of root of the susceptible tomato cultivar). According to the criteria established by Taylor (1967), the degree of resistance was classified as susceptible (S) - RI greater than $50 \%$ of the value obtained for the 'Santa Cruz Kada' tomato; Slightly resistant (SR) RI with 26 to 50\%; Moderately resistant (MoR) - RI with 11 to $25 \%$; Very resistant (VR) - RI with 1 to $10 \%$; Highly resistant (HR) - RI with less than $1 \%$, and immune (I) - when there was no reproduction.

To meet the assumptions of normality and error distribution, the data were transformed to log $(x+5)$. Data were submitted to analysis of variance, and when significant differences were identified by the F test, were grouped by the Scott-Knott test at $5 \%$ probability. For analysis, we used the statistical software AgroEstat (BARBOSA; MALDONADO JUNIOR, 2015).

\section{RESULTS AND DISCUSSION}

For both experiments the viability of the inoculum and the experimental conditions were satisfactory, since the values of TNEJ and NEJGR obtained for the susceptibility control, the 'Santa Cruz Kada' tomato, were considered as high (Tables 2 and 5), differing statistically from the other evaluated genotypes. Pinheiro et al. (2014) using the 'Rutgers' tomato as susceptibility control for a Capsicum experiment, also observed an excellent multiplication of the same nematode species, which presented high NEJGR and reproduction factor.

Tables 2 and 5 show the means of $C$. annuum genotypes, $C$. frutescens line and BRS Mari and BRS Moema cultivars inoculated with $M$. incognita race 3, M. javanica and M. enterolobii, evaluated in the first and second experiments, respectively. For all variables analyzed, in both experiments, there was a significant interaction by $\mathrm{F}$ test at $1 \%$ probability.

The $C$. frutescens line presented the lowest values for the variables analyzed in the first experiment, differing from the other evaluated 
genotypes. C. frutescens was the only genotype classified as resistant and very resistant to the three species of root-knot nematodes, as we verified $\mathrm{RF}<1$ and $\mathrm{RI}<10 \%$ (Table 2).

Table 2. Analysis of variance and test of comparison of means of the total number of eggs and second-stage juveniles (TNEJ) of root-knot nematodes, reproduction factor (RF), number of eggs and second-stage juveniles per gram of root (NEJGR), reproduction index (RI) and reaction (R) of 27 genotypes of Capsicum annuum, two commercial hot pepper cultivars, one Capsicum frutescens lineage and one cultivar 'Santa Cruz Kada' tomato.

\begin{tabular}{|c|c|c|c|c|c|c|}
\hline Genotypes (G) & TNEJ & $\mathbf{R F}$ & $\mathbf{R}^{(2)}$ & NEJGR & RI & $\mathbf{R}^{(3)}$ \\
\hline CNPH 29 & $102,733 \mathrm{c}$ & 20.54 & $\mathrm{~S}$ & $3,679.45 \mathrm{~b}$ & 49.95 & SR \\
\hline CNPH 30 & $110,600 \mathrm{c}$ & 22.12 & $\mathrm{~S}$ & $4,198.24 \mathrm{~b}$ & 58.25 & $\mathrm{~S}$ \\
\hline CNPH 31 & $107,838 \mathrm{~b}$ & 21.56 & $\mathrm{~S}$ & $2,849.89 \mathrm{c}$ & 39.18 & SR \\
\hline CNPH 32 & $118,666 \mathrm{c}$ & 23.73 & $\mathrm{~S}$ & $4,389.99 \mathrm{~b}$ & 60.31 & $\mathrm{~S}$ \\
\hline CNPH 33 & $63,733 \mathrm{~d}$ & 12.74 & $\mathrm{~S}$ & $2,290.53 \mathrm{c}$ & 30.83 & SR \\
\hline CNPH 40 & $95,413 \mathrm{~d}$ & 19.08 & $\mathrm{~S}$ & $2,200.77 \mathrm{~d}$ & 30.17 & SR \\
\hline CNPH 42 & $71,000 \mathrm{c}$ & 14.20 & $\mathrm{~S}$ & $3,450.33 \mathrm{~b}$ & 47.54 & SR \\
\hline CNPH 43 & $70,333 \mathrm{~d}$ & 14.06 & $\mathrm{~S}$ & $2,054.14 \mathrm{~d}$ & 27.34 & SR \\
\hline CNPH 44 & $97,066 \mathrm{~d}$ & 19.41 & $\mathrm{~S}$ & $2,125.13 \mathrm{e}$ & 28.67 & SR \\
\hline CNPH 45 & $141,533 b$ & 28.30 & $\mathrm{~S}$ & $2,587.84 \mathrm{c}$ & 35.36 & SR \\
\hline CNPH 47 & $55,000 \mathrm{~d}$ & 11.00 & $\mathrm{~S}$ & $1,345.85 \mathrm{e}$ & 18.26 & MoR \\
\hline CNPH 48 & $84,933 \mathrm{c}$ & 16.98 & $\mathrm{~S}$ & $2,239.33 \mathrm{c}$ & 30.68 & SR \\
\hline CNPH 66 & $146,333 \mathrm{c}$ & 29.26 & $\mathrm{~S}$ & $4,608.88 \mathrm{~b}$ & 63.97 & $\mathrm{~S}$ \\
\hline CNPH 67 & $125,333 \mathrm{~b}$ & 25.06 & $\mathrm{~S}$ & $3,959.14 b$ & 54.76 & $\mathrm{~S}$ \\
\hline CNPH 68 & $80,133 \mathrm{~d}$ & 16.02 & $\mathrm{~S}$ & $1,874.53 \mathrm{~d}$ & 25.27 & MoR \\
\hline CNPH 69 & $132,133 \mathrm{~b}$ & 26.42 & $\mathrm{~S}$ & $4,502.91 \mathrm{~b}$ & 62.29 & $\mathrm{~S}$ \\
\hline CNPH 144 & $86,466 \mathrm{~d}$ & 17.29 & $\mathrm{~S}$ & $1,674.48 \mathrm{e}$ & 22.52 & MoR \\
\hline CNPH 147 & $86,733 \mathrm{c}$ & 17.34 & $\mathrm{~S}$ & $2,152.63 \mathrm{c}$ & 29.29 & SR \\
\hline CNPH 149 & $81,800 \mathrm{~d}$ & 16.36 & $\mathrm{~S}$ & $2,745.89 \mathrm{c}$ & 37.22 & SR \\
\hline CNPH 150 & $122,000 \mathrm{~b}$ & 24.40 & $\mathrm{~S}$ & $3,578.45 b$ & 48.92 & SR \\
\hline CNPH 183 & $68,000 \mathrm{~d}$ & 13.60 & $\mathrm{~S}$ & $2,254.45 \mathrm{~d}$ & 30.90 & SR \\
\hline CNPH 184 & $100,800 \mathrm{~d}$ & 20.16 & $\mathrm{~S}$ & $2,722.89 \mathrm{c}$ & 36.64 & SR \\
\hline CNPH 185 & $131,466 \mathrm{e}$ & 26.29 & $\mathrm{~S}$ & $3,150.97 \mathrm{e}$ & 42.56 & SR \\
\hline CNPH 186 & $102,266 \mathrm{c}$ & 26.29 & $\mathrm{~S}$ & $3,740.19 b$ & 50.91 & $\mathrm{~S}$ \\
\hline CNPH 187 & $117,533 \mathrm{e}$ & 23.50 & $\mathrm{~S}$ & $2,952.30 \mathrm{~d}$ & 39.74 & SR \\
\hline CNPH 188 & $79,733 \mathrm{e}$ & 15.94 & $\mathrm{~S}$ & $1,905.14 \mathrm{e}$ & 25.68 & MoR \\
\hline CNPH 190 & $120,133 \mathrm{~d}$ & 24.02 & $\mathrm{~S}$ & $2,020.97 \mathrm{e}$ & 27.27 & SR \\
\hline C. frutescens & $3,333 \mathrm{f}$ & 0.66 & $\mathrm{R}$ & $80.22 \mathrm{f}$ & 1.06 & VR \\
\hline BRS Mari & $165,733 \mathrm{~b}$ & 33.14 & $\mathrm{~S}$ & $2,977.50 \mathrm{c}$ & 40.91 & SR \\
\hline BRS Moema & $189,733 \mathrm{~b}$ & 37.94 & $\mathrm{~S}$ & $5,292.75 b$ & 73.40 & $\mathrm{~S}$ \\
\hline 'St ${ }^{a}$ Cruz Kada' & $248,666 \mathrm{a}$ & 49.73 & $\mathrm{~S}$ & $8,065.70 \mathrm{a}$ & 100 & $\mathrm{~S}$ \\
\hline Test F & $25.96^{* * *}$ & & & $25.18^{* * *}$ & & \\
\hline \multicolumn{7}{|l|}{ Nematodes $(\mathrm{N})$} \\
\hline M. incognita race 3 & $84,162.58 \mathrm{~b}$ & 16.83 & & $2,702.43 \mathrm{~b}$ & 38.39 & \\
\hline M. javanica & $12,897.31 \mathrm{c}$ & 2.57 & & $487.28 \mathrm{c}$ & 4.89 & \\
\hline M. enterolobii & $222,990.32 \mathrm{a}$ & 44.59 & & $5,875.28 \mathrm{a}$ & 79.54 & \\
\hline Test F & $1985.31^{* *}$ & & & $1421.35^{* *}$ & & \\
\hline Interaction $(\mathrm{G} \times \mathrm{N})$ & $16.00^{* * *}$ & & & $12.80^{* *}$ & & \\
\hline $\mathrm{CV}(\%)$ & 5.50 & & & 8.89 & & \\
\hline
\end{tabular}

As expected, the cultivars BRS Mari and BRS Moema performed as resistant to $M$. javanica, with RF inferior to 1 and susceptible to $M$. enterolobii (RF>1). The cultivar BRS Moema showed a susceptibility reaction to $M$. incognita race 3 for the two experiments, both by the reproduction factor and the reproduction index (Tables 3 to 7). The resistance and susceptibility reactions of the cultivars BRS Mari and BRS Moema to the species of root-knot nematodes in the present study corroborate with the study done by Pinheiro et al. (2013). However, the cultivar BRS Mari showed susceptibility to $M$. incognita race 3 in both experiments, with RF of 33.56 (Table 3) and 63.84 (Table 6), differing from the result found by the same authors, who observed resistance of this genotype, with RF of 0.92. Probably, these differences are attributed to the populations and 
even to different $M$. incognita races used, in addition to experimental environmental methodologies and conditions. In the Pinheiro et al. (2013) study, the $M$. incognita race 1 was used and the experiment was carried out in the environmental conditions of Brasília-DF, which has an altitude of $1.200 \mathrm{~m}$ in relation to sea level, while in the present study $M$. incognita race 3 was used, and the altitude of Jaboticabal-SP is $595 \mathrm{~m}$.

Dias-Arieira et al. (2012) and AndradeJunior et al. (2016) reported that certain variations between results obtained in studies involving resistance to nematodes may occur due to differences in evaluation methodologies or to variability among the nematode isolates used in the experiments. Another characteristic that may be related to the discrepancy of the results is the environmental factor, since the studies in question were carried out under different environmental conditions.

In the first experiment, there was a significant difference between genotypes and nematode species for TNEJ and NEJGR in the F test at $1 \%$ probability (Tables 3 and 4 ). However, in the second experiment, there was no difference for $M$. enterolobii among the analyzed materials (Tables 6 and 7). The genotypes of $C$. annuum CNPH 185, CNPH 187, CNPH 188 (experiment 1) and CNPH 680, CNPH 682, CNPH 690, CNPH 693 (experiment 2) presented the lowest values of TNEJ and NEJGR for $M$. incognita race 3, being classified in distinct groups from the other genotypes analyzed by the Scott-Knott test $(\mathrm{p}<0.05)$ (Tables 3, 4, 6 and 7). When we observed the reaction of these genotypes, we classified as HR or VR by the reproduction index, however, the genotypes $\mathrm{CNPH}$ 188 and CNPH 693 were classified as susceptible by the reproduction factor, since $\mathrm{RF}>1$.

When evaluated the reaction to $M$. javanica, it was observed that 19 genotypes were classified as resistant by the reproduction factor in the first experiment (Table 3). As for experiment 2, nine genotypes were in the resistance group (Table 6). Based on RI, the genotypes CNPH 30, CNPH 40, CNPH 183, CNPH 432, CNPH 647, BRS Mari and C. frutescens were classified as $\mathrm{HR}$, with $\mathrm{RI}<1 \%$ (Tables 4 and 7). 
Table 3. Slicing of interactions between genotypes and root-knot nematodes species for total number of eggs and second-stage juveniles.

\begin{tabular}{|c|c|c|c|c|c|c|c|c|c|c|}
\hline \multirow{2}{*}{ Genotypes } & \multicolumn{3}{|c|}{ M. incognita raça 3} & \multicolumn{3}{|c|}{ M. javanica } & \multicolumn{3}{|c|}{ M. enterolobii } & \multirow{2}{*}{ Test $\mathbf{F}$} \\
\hline & TNEJ & $\mathbf{R F}$ & $\mathbf{R}^{(1)}$ & TNEJ & $\mathbf{R F}$ & $\mathbf{R}^{(\mathbf{1})}$ & TNEJ & $\mathbf{R F}$ & $\mathbf{R}^{(1)}$ & \\
\hline CNPH 29 & $49,200 \mathrm{cB}$ & 9.84 & $\mathrm{~S}$ & $4,000 \mathrm{cC}$ & 0.80 & $\mathrm{R}$ & $255,000 \mathrm{aA}$ & 51.02 & $\mathrm{~S}$ & $83.71^{* * *}$ \\
\hline CNPH 30 & $190,400 \mathrm{aA}$ & 38.08 & $\mathrm{~S}$ & $2,800 \mathrm{~dB}$ & 0.56 & $\mathrm{R}$ & $138,600 \mathrm{bA}$ & 27.72 & $\mathrm{~S}$ & $103.70^{* *}$ \\
\hline CNPH 31 & $118,800 \mathrm{bA}$ & 23.76 & $\mathrm{~S}$ & $7,916 \mathrm{bB}$ & 1.27 & $\mathrm{~S}$ & $196,800 \mathrm{bA}$ & 39.36 & $\mathrm{~S}$ & $54.77^{* *}$ \\
\hline CNPH 32 & $118,000 \mathrm{bA}$ & 23.60 & $\mathrm{~S}$ & $4,200 \mathrm{cB}$ & 0.84 & $\mathrm{R}$ & $233,800 \mathrm{bA}$ & 46.76 & $\mathrm{~S}$ & $91.67^{* *}$ \\
\hline CNPH 33 & $26,200 \mathrm{~dB}$ & 5.24 & $\mathrm{~S}$ & $7,200 \mathrm{bC}$ & 1.44 & $\mathrm{~S}$ & $157,800 \mathrm{bA}$ & 31.56 & $\mathrm{~S}$ & $34.66^{* *}$ \\
\hline CNPH 40 & $83,040 \mathrm{bA}$ & 16.61 & $\mathrm{~S}$ & $2,600 \mathrm{~dB}$ & 0.52 & $\mathrm{R}$ & $200,600 \mathrm{bA}$ & 40.12 & $\mathrm{~S}$ & $94.37^{* *}$ \\
\hline CNPH 42 & $88,400 \mathrm{bA}$ & 17.68 & $\mathrm{~S}$ & $4,000 \mathrm{cB}$ & 0.80 & $\mathrm{R}$ & $120,600 \mathrm{bA}$ & 24.12 & $\mathrm{~S}$ & $68.46^{* *}$ \\
\hline CNPH 43 & $11,000 \mathrm{eB}$ & 2.20 & $\mathrm{~S}$ & $7,200 \mathrm{bB}$ & 1.44 & $\mathrm{~S}$ & $192,800 \mathrm{aA}$ & 38.56 & $\mathrm{~S}$ & $63.99^{* *}$ \\
\hline CNPH 44 & $10,600 \mathrm{eB}$ & 2.12 & $\mathrm{~S}$ & $4,000 \mathrm{cC}$ & 0.80 & $\mathrm{R}$ & $276,600 \mathrm{aA}$ & 55.32 & $\mathrm{~S}$ & $92.58^{* *}$ \\
\hline CNPH 45 & $113,400 \mathrm{bB}$ & 22.68 & $\mathrm{~S}$ & $5,400 \mathrm{cC}$ & 1.08 & $\mathrm{~S}$ & $305,800 \mathrm{aA}$ & 61.16 & $\mathrm{~S}$ & $84.74^{* *}$ \\
\hline CNPH 47 & $32,000 \mathrm{cB}$ & 6.40 & $\mathrm{~S}$ & $5,400 \mathrm{cC}$ & 1.08 & $\mathrm{~S}$ & $127,600 \mathrm{bB}$ & 25.52 & $\mathrm{~S}$ & $44.93^{* *}$ \\
\hline CNPH 48 & $82,000 \mathrm{bA}$ & 16.40 & $\mathrm{~S}$ & $5,600 \mathrm{bB}$ & 1.12 & $\mathrm{~S}$ & $167,200 \mathrm{bA}$ & 33.44 & $\mathrm{~S}$ & $52.75^{* *}$ \\
\hline CNPH 66 & $185,000 \mathrm{aA}$ & 37.01 & $\mathrm{~S}$ & $2,800 \mathrm{~dB}$ & 0.56 & $\mathrm{R}$ & $251,200 \mathrm{aA}$ & 50.24 & $\mathrm{~S}$ & $120.33^{\text {*** }}$ \\
\hline CNPH 67 & $201,400 \mathrm{aA}$ & 40.28 & $\mathrm{~S}$ & $3,600 \mathrm{cB}$ & 0.72 & $\mathrm{R}$ & $171,000 \mathrm{bA}$ & 34.20 & $\mathrm{~S}$ & $92.06^{* *}$ \\
\hline CNPH 68 & $22,200 \mathrm{~dB}$ & 4.44 & $\mathrm{~S}$ & $5,000 \mathrm{cC}$ & 1.00 & $\mathrm{~S}$ & $213,200 \mathrm{aA}$ & 42.62 & $\mathrm{~S}$ & $72.33^{* *}$ \\
\hline CNPH 69 & $210,200 \mathrm{aA}$ & 42.05 & $\mathrm{~S}$ & $4,600 \mathrm{cB}$ & 0.92 & $\mathrm{R}$ & $181,600 \mathrm{bA}$ & 36.32 & $\mathrm{~S}$ & $88.43^{* *}$ \\
\hline CNPH 144 & $17,000 \mathrm{~dB}$ & 3.40 & $\mathrm{~S}$ & $7,000 \mathrm{bC}$ & 140 & $\mathrm{~S}$ & $235,400 \mathrm{aA}$ & 47.08 & $\mathrm{~S}$ & $58.05^{* *}$ \\
\hline CNPH 147 & $63,800 \mathrm{cB}$ & 12.76 & $\mathrm{~S}$ & $4,800 \mathrm{cC}$ & 0.96 & $\mathrm{R}$ & $191,600 \mathrm{bA}$ & 38.32 & $\mathrm{~S}$ & $63.87^{* *}$ \\
\hline CNPH 149 & $25,600 \mathrm{~dB}$ & 5.12 & $\mathrm{~S}$ & $4,800 \mathrm{cC}$ & 0.96 & $\mathrm{R}$ & $215,000 \mathrm{aA}$ & 43.01 & $\mathrm{~S}$ & $74.88^{* *}$ \\
\hline CNPH 150 & $119,200 \mathrm{bB}$ & 23.84 & $\mathrm{~S}$ & $6,600 \mathrm{bC}$ & 1.32 & $\mathrm{~S}$ & $240,200 \mathrm{aA}$ & 48.14 & $\mathrm{~S}$ & $70.04^{* *}$ \\
\hline CNPH 183 & $79,000 \mathrm{bA}$ & 15.80 & $\mathrm{~S}$ & $1,400 \mathrm{~dB}$ & 0.28 & $\mathrm{R}$ & $123,600 \mathrm{bA}$ & 24.72 & $\mathrm{~S}$ & $108.42^{* *}$ \\
\hline CNPH 184 & $13,200 \mathrm{eB}$ & 2.64 & $\mathrm{~S}$ & $7,800 \mathrm{bB}$ & 1.32 & $\mathrm{~S}$ & $281,400 \mathrm{aA}$ & 5630 & $\mathrm{~S}$ & $69.04^{* *}$ \\
\hline CNPH 185 & $3,800 \mathrm{fB}$ & 0.76 & $\mathrm{R}$ & $2,200 \mathrm{~dB}$ & 0.44 & $\mathrm{R}$ & $388,400 \mathrm{aA}$ & 77.66 & $\mathrm{~S}$ & $151.28^{* *}$ \\
\hline CNPH 186 & $51,000 \mathrm{cB}$ & 10.20 & $\mathrm{~S}$ & $4,600 \mathrm{cC}$ & 0.92 & $\mathrm{R}$ & $251,200 \mathrm{aA}$ & 50.21 & $\mathrm{~S}$ & $81.26^{* *}$ \\
\hline CNPH 187 & $3,600 \mathrm{fB}$ & 0.72 & $\mathrm{R}$ & $4,800 \mathrm{cB}$ & 0.96 & $\mathrm{R}$ & $344,200 \mathrm{aA}$ & 68.84 & $\mathrm{~S}$ & $125.40^{* *}$ \\
\hline CNPH 188 & $5,600 \mathrm{fB}$ & 1.12 & $\mathrm{~S}$ & $3,800 \mathrm{cB}$ & 0.76 & $\mathrm{R}$ & $229,800 \mathrm{aA}$ & 45.96 & $\mathrm{~S}$ & $98.51^{* *}$ \\
\hline CNPH 190 & $17,800 \mathrm{~dB}$ & 3.56 & $\mathrm{~S}$ & $5,000 \mathrm{cC}$ & 1.00 & $\mathrm{~S}$ & $337,600 \mathrm{aA}$ & 67.49 & $\mathrm{~S}$ & $95.90^{* *}$ \\
\hline C. frutescens & $4,200 \mathrm{fA}$ & 0.84 & $\mathrm{R}$ & $1,800 \mathrm{~dB}$ & 0.36 & $\mathrm{R}$ & $4,000 \mathrm{cA}$ & 0.80 & $\mathrm{R}$ & $4.40^{*}$ \\
\hline BRS Mari & $167,800 \mathrm{aB}$ & 33.56 & $\mathrm{~S}$ & $4,000 \mathrm{cC}$ & 080 & $\mathrm{R}$ & $325,400 \mathrm{aA}$ & 65.08 & $\mathrm{~S}$ & $110.53^{* *}$ \\
\hline BRS Moema & $264,400 \mathrm{aA}$ & 52.88 & S & $4,200 \mathrm{cB}$ & 0.84 & $\mathrm{R}$ & $300,600 \mathrm{aA}$ & 60.10 & $\mathrm{~S}$ & $110.08^{* *}$ \\
\hline 'St ${ }^{\mathrm{a}}$ Cruz Kada' & $231,200 \mathrm{aA}$ & 46.24 & $\mathrm{~S}$ & $26,070 \mathrm{aA}$ & 52.14 & $\mathrm{~S}$ & $254,100 \mathrm{aA}$ & 51.00 & $\mathrm{~S}$ & $0.17^{\mathrm{ns}}$ \\
\hline Test $\mathrm{F}$ & $32.64^{*}$ & & & $13.67^{* * *}$ & & & $11.65^{* *}$ & & & \\
\hline
\end{tabular}

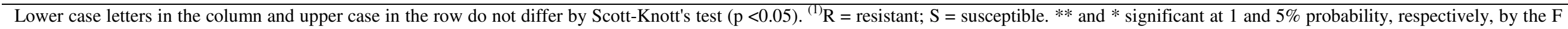
test. 
Table 4. Slicing of interactions between genotypes and root-knot nematodes species for number of eggs and second-stage juveniles per gram of root.

\begin{tabular}{|c|c|c|c|c|c|c|c|c|c|c|}
\hline \multirow{2}{*}{ Genotypes } & \multicolumn{3}{|c|}{ M. incognita raça 3} & \multicolumn{3}{|l|}{ M. javanica } & \multicolumn{3}{|c|}{ M. enterolobii } & \multirow[t]{2}{*}{ Test $\mathrm{F}$} \\
\hline & NEJGR & RI & $\mathbf{R}^{(\mathbf{1})}$ & NEJGR & RI & $\mathbf{R}^{(\mathbf{1})}$ & NEJGR & $\mathbf{R I}$ & $\mathbf{R}^{(\mathbf{1})}$ & \\
\hline CNPH 29 & $1,811.72 \mathrm{Bb}$ & 25.74 & MoR & $217.52 \mathrm{bC}$ & 2.18 & VR & $9,586.62 \mathrm{aA}$ & 129.80 & $\mathrm{~S}$ & $56.70^{* *}$ \\
\hline CNPH 30 & $6,835.21 \mathrm{aA}$ & 97.11 & $\mathrm{~S}$ & $9108 \mathrm{cB}$ & 0.91 & HR & $5,668.45 \mathrm{aA}$ & 76.75 & $\mathrm{~S}$ & $96.25^{* *}$ \\
\hline CNPH 31 & $3,594.87 \mathrm{bA}$ & 51.07 & $S$ & $135.22 \mathrm{bB}$ & 1.36 & VR & $4,785.73 \mathrm{aA}$ & 64.80 & $\mathrm{~S}$ & $51.41^{* *}$ \\
\hline CNPH 32 & $4,902.09 \mathrm{aA}$ & 69.65 & $S$ & $186.85 \mathrm{bB}$ & 1.88 & VR & $8,081.05 \mathrm{aA}$ & 109.41 & $\mathrm{~S}$ & $71.60^{* * *}$ \\
\hline CNPH 33 & $768.30 \mathrm{cB}$ & 10.92 & VR & $302.52 \mathrm{bC}$ & 3.04 & VR & $5,800.79 \mathrm{aA}$ & 78.54 & $\mathrm{~S}$ & $33.08^{* *}$ \\
\hline CNPH 40 & 2,193.66 bA & 31.17 & SR & $84.48 \mathrm{cB}$ & 0.85 & HR & $4,362.47 \mathrm{aA}$ & 59.06 & $\mathrm{~S}$ & $68.16^{* *}$ \\
\hline CNPH 42 & $4,771.14 \mathrm{aA}$ & 67.79 & S & $198.10 \mathrm{bB}$ & 1.99 & VR & $5,448.87 \mathrm{aA}$ & 73.77 & $\mathrm{~S}$ & $56.98^{* *}$ \\
\hline CNPH 43 & $314.62 \mathrm{eB}$ & 4.47 & VR & $456.56 \mathrm{bB}$ & 4.59 & VR & $5,391.25 \mathrm{aA}$ & 72.99 & $\mathrm{~S}$ & $43.32^{\text {** }}$ \\
\hline CNPH 44 & $215.06 \mathrm{eB}$ & 3.06 & VR & $129.25 \mathrm{bB}$ & 1.30 & VR & $6,031.10 \mathrm{aA}$ & 81.66 & $\mathrm{~S}$ & $70.11^{\text {** }}$ \\
\hline CNPH 45 & $2,210.77 \mathrm{bB}$ & 31.41 & SR & $142.41 \mathrm{bC}$ & 1.43 & VR & $5,410.36 \mathrm{aA}$ & 73.25 & $\mathrm{~S}$ & $59.11^{* *}$ \\
\hline CNPH 47 & $903.97 \mathrm{cB}$ & 12.84 & MoR & $139.76 \mathrm{bC}$ & 1.40 & VR & $2,993.82 \mathrm{aA}$ & 40.53 & SR & $34.52^{* * *}$ \\
\hline CNPH 48 & $2,539.68 \mathrm{bA}$ & 36.08 & SR & $169.89 \mathrm{bB}$ & 1.71 & VR & $4,008.43 \mathrm{aA}$ & 54.27 & $\mathrm{~S}$ & $3977^{* * *}$ \\
\hline CNPH 66 & $7,763.23 \mathrm{aA}$ & 110.35 & $\mathrm{~S}$ & $136.38 \mathrm{cB}$ & 1.37 & VR & $5,923.00 \mathrm{aA}$ & 80.19 & $\mathrm{~S}$ & $82.66^{* * *}$ \\
\hline CNPH 67 & $6,093.32 \mathrm{aA}$ & 86.57 & $S$ & $173.78 \mathrm{bB}$ & 1.75 & VR & $5,614.90 \mathrm{aA}$ & 76.02 & $\mathrm{~S}$ & $64.02^{* *}$ \\
\hline CNPH 68 & $629.45 \mathrm{cB}$ & 8.94 & VR & $207.68 \mathrm{bC}$ & 2.09 & VR & $4,824.67 \mathrm{aA}$ & 54.44 & $\mathrm{~S}$ & $47.14^{* *}$ \\
\hline CNPH 69 & $7,064.34 \mathrm{aA}$ & 100.87 & $S$ & $216.12 \mathrm{bB}$ & 2.17 & VR & $6,192.52 \mathrm{aA}$ & 83.84 & $\mathrm{~S}$ & $66.14^{* *}$ \\
\hline CNPH 144 & $335.51 \mathrm{eB}$ & 4.77 & VR & $193.33 \mathrm{bB}$ & 1.94 & VR & $4,494.60 \mathrm{aA}$ & 60.85 & $\mathrm{~S}$ & $45.15^{* *}$ \\
\hline CNPH 147 & $1,732.85 \mathrm{bA}$ & 24.62 & MoR & $204.57 \mathrm{bB}$ & 2.05 & VR & $4,520.49 \mathrm{aA}$ & 61.20 & $\mathrm{~S}$ & $35.95^{* *}$ \\
\hline CNPH 149 & $1,481.41 \mathrm{bB}$ & 21.05 & MoR & $243.95 \mathrm{bC}$ & 2.45 & VR & $6,656.21 \mathrm{aA}$ & 90.12 & $\mathrm{~S}$ & $48.47^{* *}$ \\
\hline CNPH 150 & $3,246.88 \mathrm{bB}$ & 46.13 & SR & $216.41 \mathrm{bC}$ & 2.17 & VR & $7,517.64 \mathrm{aA}$ & 101.78 & $\mathrm{~S}$ & $55.73^{* *}$ \\
\hline CNPH 183 & $1,951.24 \mathrm{bB}$ & 27.72 & SR & $50.66 \mathrm{dC}$ & 0.51 & HR & $4,858.10 \mathrm{aA}$ & 65.78 & $\mathrm{~S}$ & $89.20^{* * *}$ \\
\hline CNPH 184 & $389.21 \mathrm{~dB}$ & 5.53 & VR & $225.94 \mathrm{bB}$ & 2.27 & VR & $7,601.03 \mathrm{aA}$ & 102.91 & $\mathrm{~S}$ & $53.10^{* *}$ \\
\hline CNPH 185 & $13 ., 39 \mathrm{fB}$ & 1.97 & VR & $111.42 \mathrm{cB}$ & 1.12 & VR & $9,514.54 \mathrm{aA}$ & 128.82 & $\mathrm{~S}$ & $96.74^{* *}$ \\
\hline CNPH 186 & $2,404.56 \mathrm{bB}$ & 34.16 & SR & $220.40 \mathrm{bC}$ & 2.21 & VR & $8,900.20 \mathrm{aA}$ & 120.50 & $\mathrm{~S}$ & $55.92^{* *}$ \\
\hline CNPH 187 & $236.55 \mathrm{eB}$ & 3.36 & VR & $238.43 \mathrm{bB}$ & 2.39 & VR & $8,381.94 \mathrm{aA}$ & 113.49 & $\mathrm{~S}$ & $69.76^{* *}$ \\
\hline CNPH 188 & $226.76 \mathrm{eB}$ & 3.22 & VR & $134.97 \mathrm{bB}$ & 1.36 & VR & $5,353.72 \mathrm{aA}$ & 72.49 & $\mathrm{~S}$ & $67.43^{* *}$ \\
\hline CNPH 190 & $385.65 \mathrm{~dB}$ & 5.48 & VR & $145.56 \mathrm{bC}$ & 1.46 & VR & $5,549.07 \mathrm{aA}$ & 75.13 & $\mathrm{~S}$ & $60.48^{* * *}$ \\
\hline C. frutescens & $113.01 \mathrm{fA}$ & 1.61 & VR & $36.35 \mathrm{cB}$ & 0.37 & HR & $91.32 \mathrm{bA}$ & 1.24 & VR & $4.37^{*}$ \\
\hline BRS Mari & $3,129.14 \mathrm{bA}$ & 44.46 & SR & $82.16 \mathrm{cB}$ & 0.83 & HR & $5,721.22 \mathrm{aA}$ & 77.46 & $\mathrm{~S}$ & $84.80^{* *}$ \\
\hline BRS Moema & $8,414.47 \mathrm{aA}$ & 119.55 & $S$ & $108.24 \mathrm{cB}$ & 1.09 & VR & $7,395.13 \mathrm{aA}$ & 100.12 & $\mathrm{~S}$ & $96.59^{\text {*** }}$ \\
\hline 'St ${ }^{\mathrm{a}}$ Cruz Kada' & $6,382.30 \mathrm{aA}$ & 100.00 & $S$ & $9,956.70 \mathrm{aA}$ & 100.00 & S & $7,386 \mathrm{aA}$ & 100.00 & $\mathrm{~S}$ & $0.75^{\mathrm{ns}}$ \\
\hline Test $\mathrm{F}$ & $28.15^{* *}$ & & & $12.57^{* *}$ & & & $10.07^{* *}$ & & & \\
\hline
\end{tabular}

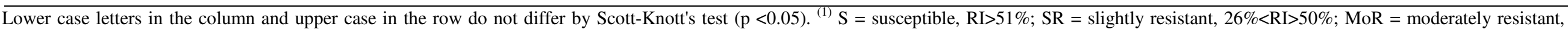
$11 \%<\mathrm{RI}>25 \%$; VR $=$ very resistant, $1 \%<\mathrm{RI}>10 \%$; HR = highly resistant, $\mathrm{IR}<1 \%$. $* *$ and $*$ significant at 1 and $5 \%$ probability, respectively, ${ }^{\mathrm{ns}}$ Not significant, by the $\mathrm{F}$ test. 
Table 5. Analysis of variance and test of comparison of means of the total number of eggs and second-stage juveniles (TNEJ) of root-knot nematodes, reproduction factor (RF), number of eggs and second-stage juveniles per gram of root (NEJGR), reproduction index (RI) and reaction (R) of 36 genotypes of Capsicum annuum, two commercial hot pepper cultivars and one cultivar 'Santa Cruz Kada' tomato.

\begin{tabular}{|c|c|c|c|c|c|c|}
\hline Genotypes (G) & TNEJ & $\mathbf{R F}$ & $\mathbf{R}^{(2)}$ & NEJGR & RI & $\mathbf{R}^{(3)}$ \\
\hline CNPH 64 & $97,733 \mathrm{c}^{(1)}$ & 19.54 & $\mathrm{~S}$ & $4,915.31 \mathrm{~d}$ & 36.41 & SR \\
\hline CNPH 145 & $111,544 \mathrm{c}$ & 22.30 & $\mathrm{~S}$ & $3,731.21 \mathrm{e}$ & 26.57 & SR \\
\hline CNPH 191 & $178,122 \mathrm{~b}$ & 35.62 & $\mathrm{~S}$ & $6,442.23 \mathrm{c}$ & 34.46 & SR \\
\hline CNPH 194 & $216,333 \mathrm{~b}$ & 43.26 & $\mathrm{~S}$ & $6,605.61 \mathrm{c}$ & 42.15 & SR \\
\hline CNPH 198 & $197,566 \mathrm{~b}$ & 39.51 & $\mathrm{~S}$ & $9,105.82 \mathrm{~b}$ & 61.82 & $\mathrm{~S}$ \\
\hline CNPH 199 & $234,122 \mathrm{~b}$ & 46.82 & $\mathrm{~S}$ & $8,181.95 \mathrm{~d}$ & 49.80 & SR \\
\hline CNPH 200 & $118,822 \mathrm{~b}$ & 23.76 & $\mathrm{~S}$ & $3,983.15 \mathrm{~d}$ & 27.06 & SR \\
\hline CNPH 291 & $230,133 \mathrm{~b}$ & 46.02 & $\mathrm{~S}$ & $9,382.83 \mathrm{c}$ & 53.09 & $\mathrm{~S}$ \\
\hline CNPH 292 & $200,066 \mathrm{~b}$ & 40.01 & $\mathrm{~S}$ & $7,380.24 \mathrm{c}$ & 43.35 & SR \\
\hline CNPH 295 & $178,888 \mathrm{~b}$ & 35.77 & $\mathrm{~S}$ & $5,184.28 \mathrm{c}$ & 35.74 & SR \\
\hline CNPH 296 & $241,288 \mathrm{~b}$ & 48.25 & $\mathrm{~S}$ & $6,939.06 \mathrm{c}$ & 40.75 & SR \\
\hline CNPH 297 & $183,444 \mathrm{~b}$ & 36.68 & $\mathrm{~S}$ & $5,961.58 \mathrm{c}$ & 36.59 & SR \\
\hline CNPH 432 & $271,400 \mathrm{c}$ & 54.28 & $\mathrm{~S}$ & $7,644.21 \mathrm{e}$ & 39.82 & SR \\
\hline CNPH 433 & $180,100 \mathrm{~b}$ & 36.02 & $\mathrm{~S}$ & $4,633.18 \mathrm{~d}$ & 26.62 & SR \\
\hline CNPH 580 & $215,288 \mathrm{~b}$ & 43.05 & $\mathrm{~S}$ & $5,568.14 \mathrm{~d}$ & 30.98 & SR \\
\hline CNPH 581 & $194,177 \mathrm{~b}$ & 38.83 & $\mathrm{~S}$ & $6,387.96 \mathrm{c}$ & 33.87 & SR \\
\hline CNPH 582 & $206,800 \mathrm{c}$ & 41.36 & $\mathrm{~S}$ & $5,751.49 \mathrm{e}$ & 31.84 & SR \\
\hline CNPH 583 & $178,244 \mathrm{c}$ & 35.64 & $\mathrm{~S}$ & $5,844.53 \mathrm{e}$ & 34.16 & SR \\
\hline CNPH 593 & $174,766 \mathrm{~b}$ & 34.95 & $\mathrm{~S}$ & $4,814.48 \mathrm{~d}$ & 27.22 & SR \\
\hline CNPH 602 & $193,188 \mathrm{~b}$ & 38.63 & $\mathrm{~S}$ & $6,518.16 \mathrm{c}$ & 37.70 & SR \\
\hline CNPH 640 & $175,222 \mathrm{c}$ & 35.04 & $\mathrm{~S}$ & $5,146.33 \mathrm{e}$ & 25.06 & MoR \\
\hline CNPH 641 & $116,344 \mathrm{~b}$ & 23.26 & $\mathrm{~S}$ & $5,687.13 \mathrm{c}$ & 34.56 & SR \\
\hline CNPH 642 & $123,122 \mathrm{c}$ & 24.62 & $\mathrm{~S}$ & $8,590.06 \mathrm{c}$ & 44.38 & SR \\
\hline CNPH 644 & $223,077 \mathrm{~b}$ & 44.61 & $\mathrm{~S}$ & $9,225.24 \mathrm{~b}$ & 56.98 & $\mathrm{~S}$ \\
\hline CNPH 646 & $197,477 \mathrm{c}$ & 39.49 & $\mathrm{~S}$ & $8,258.64 \mathrm{e}$ & 41.72 & SR \\
\hline CNPH 677 & $87,711 \mathrm{c}$ & 17.54 & $\mathrm{~S}$ & $2,176.90 \mathrm{e}$ & 14.83 & MoR \\
\hline CNPH 680 & $116,466 \mathrm{~d}$ & 23.29 & $\mathrm{~S}$ & $3,293.04 \mathrm{~g}$ & 24.83 & MoR \\
\hline CNPH 682 & $123,388 \mathrm{~d}$ & 24.67 & $\mathrm{~S}$ & $3,28 ., 82 \mathrm{f}$ & 24.93 & MoR \\
\hline CNPH 683 & $138,644 \mathrm{c}$ & 27.72 & $\mathrm{~S}$ & $4,385.34 \mathrm{e}$ & 27.78 & SR \\
\hline CNPH 684 & $54,211 \mathrm{c}$ & 10.84 & $\mathrm{~S}$ & $2,351.64 \mathrm{e}$ & 15.28 & MoR \\
\hline CNPH 687 & $156,977 \mathrm{~b}$ & 31.39 & $\mathrm{~S}$ & $7,460.63 \mathrm{c}$ & 43.96 & SR \\
\hline CNPH 688 & $176,944 \mathrm{~b}$ & 35.38 & $\mathrm{~S}$ & $6,163.10 \mathrm{c}$ & 41.59 & SR \\
\hline CNPH 690 & $53,477 \mathrm{~d}$ & 10.69 & $\mathrm{~S}$ & $1,986.06 \mathrm{f}$ & 14.87 & MoR \\
\hline CNPH 691 & $84,711 \mathrm{c}$ & 16.94 & $\mathrm{~S}$ & $3,660.79 \mathrm{e}$ & 27.66 & SR \\
\hline CNPH 692 & $133,800 \mathrm{~b}$ & 26.76 & $\mathrm{~S}$ & $5,531.69 \mathrm{c}$ & 31.41 & SR \\
\hline CNPH 693 & $84,422 \mathrm{~d}$ & 16.88 & $\mathrm{~S}$ & $2,136.56 \mathrm{f}$ & 15.81 & MoR \\
\hline BRS Mari & $196,088 \mathrm{c}$ & 39.21 & $\mathrm{~S}$ & $4,410.42 \mathrm{e}$ & 22.84 & MoR \\
\hline BRS Moema & $202,944 \mathrm{~b}$ & 40.58 & $\mathrm{~S}$ & $6,816.15 \mathrm{~d}$ & 39.75 & SR \\
\hline 'St' Cruz Kada' & $721,444 \mathrm{a}$ & 144.28 & $\mathrm{~S}$ & $16,800.63 \mathrm{a}$ & 100.00 & $\mathrm{~S}$ \\
\hline Test F & $12.62^{* *}$ & & & $21.85^{* *}$ & & \\
\hline \multicolumn{7}{|l|}{ Nematodes (N) } \\
\hline M. incognita raça 3 & $220507.69 \mathrm{~b}$ & 44.10 & & $7801.28 \mathrm{~b}$ & 29.84 & \\
\hline M. javanica & $\begin{array}{l}33955.55 \mathrm{c} \\
281576.07\end{array}$ & $\begin{array}{l}6.79 \\
56.31\end{array}$ & & $1002.59 \mathrm{c}$ & $\begin{array}{l}9.07 \\
68.65\end{array}$ & \\
\hline Test F & $652.44^{* *}$ & & & $1313.90^{* *}$ & & \\
\hline Interaction $(\mathrm{G} \times \mathrm{N})$ & $9.58^{* *}$ & & & $14.96^{* *}$ & & \\
\hline $\mathrm{CV}(\%)$ & 10.02 & & & 9.47 & & \\
\hline
\end{tabular}


Table 6. Slicing of interactions between genotypes and root-knot nematodes species for total number of eggs and second-stage juveniles.

\begin{tabular}{|c|c|c|c|c|c|c|c|c|c|c|}
\hline \multirow{2}{*}{ Genotypes } & \multicolumn{3}{|c|}{ M. incognita raça 3} & \multicolumn{3}{|l|}{ M.javanica } & \multicolumn{3}{|c|}{ M. enterolobii } & \multirow[t]{2}{*}{ Test $\mathbf{F}$} \\
\hline & TNEJ & RF & $\mathbf{R}^{(\mathbf{1})}$ & TNEJ & RF & $\mathbf{R}^{(1)}$ & TNEJ & RF & $\mathbf{R}^{(\mathbf{1})}$ & \\
\hline CNPH 64 & $13,200 \mathrm{cB}$ & 2.64 & $\mathrm{~S}$ & $28,533 \mathrm{cB}$ & 5.70 & $\mathrm{~S}$ & 251,466 aA & 50.29 & $\mathrm{~S}$ & $12.01^{* *}$ \\
\hline CNPH 145 & $42,900 \mathrm{bB}$ & 8.58 & S & $13,066 \mathrm{cB}$ & 2.61 & $\mathrm{~S}$ & $278,666 \mathrm{aA}$ & 55.73 & $\mathrm{~S}$ & $11.68^{* *}$ \\
\hline CNPH 191 & $255,300 \mathrm{aA}$ & 51.06 & $\mathrm{~S}$ & $25,333 \mathrm{cB}$ & 5.06 & $\mathrm{~S}$ & $253,733 \mathrm{aA}$ & 50.74 & $\mathrm{~S}$ & $8.63^{* *}$ \\
\hline CNPH 194 & $261,000 \mathrm{aA}$ & 52.20 & $\mathrm{~S}$ & $60,800 \mathrm{bB}$ & 12.16 & $\mathrm{~S}$ & $327,200 \mathrm{aA}$ & 65.44 & $\mathrm{~S}$ & $4.57^{*}$ \\
\hline CNPH 198 & $176,700 \mathrm{aA}$ & 35.34 & $\mathrm{~S}$ & $137,066 \mathrm{bA}$ & 27.41 & $\mathrm{~S}$ & $278,933 \mathrm{aA}$ & 55.78 & $\mathrm{~S}$ & $0.64^{\mathrm{ns}}$ \\
\hline CNPH 199 & $309,300 \mathrm{aA}$ & 61.86 & $\mathrm{~S}$ & $7.200 \mathrm{~dB}$ & 1.44 & $\mathrm{~S}$ & $385,866 \mathrm{aA}$ & 77.17 & $\mathrm{~S}$ & $27.19^{* *}$ \\
\hline CNPH 200 & $83,400 \mathrm{bB}$ & 16.68 & $\mathrm{~S}$ & $20,000 \mathrm{cB}$ & 4.00 & $\mathrm{~S}$ & 253,066 aA & 50.61 & $\mathrm{~S}$ & $8.11^{* *}$ \\
\hline CNPH 291 & $352,800 \mathrm{aA}$ & 72.72 & $\mathrm{~S}$ & $8,800 \mathrm{~dB}$ & 1.76 & $\mathrm{~S}$ & $328,800 \mathrm{aA}$ & 65.76 & $\mathrm{~S}$ & $22.61^{* *}$ \\
\hline CNPH 292 & $287,400 \mathrm{aA}$ & 57.48 & $\mathrm{~S}$ & $21,066 \mathrm{cB}$ & 4.21 & $\mathrm{~S}$ & $291,733 \mathrm{aA}$ & 58.34 & $\mathrm{~S}$ & $11.11^{* *}$ \\
\hline CNPH 295 & $114,000 \mathrm{bB}$ & 22.80 & $\mathrm{~S}$ & $85,333 \mathrm{bB}$ & 17.06 & $\mathrm{~S}$ & 337,333 aA & 67.46 & $\mathrm{~S}$ & $3.00^{\mathrm{ns}}$ \\
\hline CNPH 296 & $421,200 \mathrm{aA}$ & 84.24 & $\mathrm{~S}$ & $14,400 \mathrm{cB}$ & 2.88 & $\mathrm{~S}$ & 288,266 aA & 57.65 & S & $16.68^{* *}$ \\
\hline CNPH 297 & $229,800 \mathrm{aA}$ & 45.96 & $\mathrm{~S}$ & $15,200 \mathrm{cB}$ & 3.04 & $\mathrm{~S}$ & $305,333 \mathrm{aA}$ & 61.06 & $\mathrm{~S}$ & $14.01^{* *}$ \\
\hline CNPH 432 & $442,200 \mathrm{aA}$ & 88.44 & $\mathrm{~S}$ & $1,600 \mathrm{fB}$ & 0.32 & $\mathrm{R}$ & $370,400 \mathrm{aA}$ & 74.08 & $\mathrm{~S}$ & $108.05^{* *}$ \\
\hline CNPH 433 & $319,500 \mathrm{aA}$ & 63.90 & $\mathrm{~S}$ & $11,466 \mathrm{cB}$ & 2.29 & $\mathrm{~S}$ & $209,333 \mathrm{aA}$ & 41.86 & $\mathrm{~S}$ & $15.90^{* *}$ \\
\hline CNPH 580 & $331,200 \mathrm{aA}$ & 66.24 & S & $8,266 \mathrm{~dB}$ & 1.65 & S & $306,400 \mathrm{aA}$ & 61.28 & S & $22.09^{* *}$ \\
\hline CNPH 581 & $363,600 \mathrm{aA}$ & 72.72 & $S$ & $11,466 \mathrm{cB}$ & 2.29 & $\mathrm{~S}$ & 207,466 aA & 41.49 & $\mathrm{~S}$ & $17.08^{* *}$ \\
\hline CNPH 582 & $332,400 \mathrm{aA}$ & 66.48 & $S$ & $6,133 \mathrm{eB}$ & 1.22 & $\mathrm{~S}$ & 281,866 aA & 56.37 & $\mathrm{~S}$ & $58.79^{* *}$ \\
\hline CNPH 583 & $252,600 \mathrm{aA}$ & 50.52 & $\mathrm{~S}$ & $4,533 \mathrm{eB}$ & 0.90 & $\mathrm{R}$ & $277,600 \mathrm{aA}$ & 55.52 & $\mathrm{~S}$ & $61.13^{* *}$ \\
\hline CNPH 593 & $266,700 \mathrm{aA}$ & 53.34 & $\mathrm{~S}$ & $8,000 \mathrm{~dB}$ & 1.60 & S & $249.600 \mathrm{aA}$ & 49.92 & $\mathrm{~S}$ & $22.05^{* *}$ \\
\hline CNPH 602 & $272,100 \mathrm{aA}$ & 54.42 & $\mathrm{~S}$ & $11,466 \mathrm{cB}$ & 2.29 & $\mathrm{~S}$ & $296,000 \mathrm{aA}$ & 59.20 & S & $16.86^{* *}$ \\
\hline CNPH 640 & $327,000 \mathrm{aA}$ & 65.40 & $\mathrm{~S}$ & $2,933 \mathrm{eB}$ & 0.58 & $\mathrm{R}$ & $195,733 \mathrm{aA}$ & 39.14 & $\mathrm{~S}$ & $49.43^{* *}$ \\
\hline CNPH 641 & $155,700 \mathrm{aA}$ & 31.14 & $S$ & $15,466 \mathrm{cB}$ & 3.09 & $\mathrm{~S}$ & $177,866 \mathrm{aA}$ & 35.57 & S & $9.61^{* *}$ \\
\hline CNPH 642 & $247,500 \mathrm{aA}$ & 49.50 & $\mathrm{~S}$ & $5,333 \mathrm{~dB}$ & 1.06 & $\mathrm{~S}$ & $116,533 \mathrm{aA}$ & 23.30 & $\mathrm{~S}$ & $20.97^{* *}$ \\
\hline CNPH 644 & $260,700 \mathrm{aA}$ & 52.14 & $\mathrm{~S}$ & $78,933 \mathrm{bB}$ & 15.78 & $\mathrm{~S}$ & $329,600 \mathrm{aA}$ & 65.92 & S & $2.88^{\mathrm{ns}}$ \\
\hline CNPH 646 & $344,700 \mathrm{aA}$ & 68.94 & $\mathrm{~S}$ & $3,200 \mathrm{fB}$ & 0.64 & $\mathrm{R}$ & $244,533 \mathrm{aA}$ & 48.90 & $\mathrm{~S}$ & $90.51^{* *}$ \\
\hline CNPH 677 & $49,800 \mathrm{bB}$ & 9.96 & $\mathrm{~S}$ & $5,600 \mathrm{dC}$ & 1.12 & $\mathrm{~S}$ & $207,733 \mathrm{aA}$ & 41.54 & $\mathrm{~S}$ & $16.78^{* *}$ \\
\hline CNPH 680 & $3,000 \mathrm{cB}$ & 0.60 & $\mathrm{R}$ & 3,466 eB & 0.69 & $\mathrm{R}$ & $342,933 \mathrm{aA}$ & 68.58 & S & $71.74^{* *}$ \\
\hline CNPH 682 & $2,700 \mathrm{cB}$ & 0.54 & $\mathrm{R}$ & $9,866 \mathrm{cC}$ & 1.97 & $\mathrm{~S}$ & $357,600 \mathrm{aA}$ & 71.52 & $\mathrm{~S}$ & $43.67^{* *}$ \\
\hline CNPH 683 & $102,600 \mathrm{bA}$ & 20.52 & S & $2,666 \mathrm{eB}$ & 0.53 & $\mathrm{R}$ & $310,666 \mathrm{aA}$ & 62.13 & S & $45.82^{* *}$ \\
\hline CNPH 684 & $50,100 \mathrm{bA}$ & 10.02 & $\mathrm{~S}$ & $4,800 \mathrm{~dB}$ & 0.96 & $\mathrm{R}$ & $107,733 \mathrm{aA}$ & 21.54 & $\mathrm{~S}$ & $14.13^{* *}$ \\
\hline CNPH 687 & $172,800 \mathrm{aA}$ & 34.56 & $\mathrm{~S}$ & $8,533 \mathrm{~dB}$ & 1.70 & $\mathrm{~S}$ & $289,600 \mathrm{aA}$ & 57.92 & $\mathrm{~S}$ & $18.93^{* *}$ \\
\hline CNPH 688 & $97,500 \mathrm{bB}$ & 19.50 & $S$ & $39,733 \mathrm{bB}$ & 7.94 & $\mathrm{~S}$ & $393,600 \mathrm{aA}$ & 78.72 & S & $6.80^{* *}$ \\
\hline CNPH 690 & $3,900 \mathrm{dC}$ & 0.78 & $\mathrm{R}$ & $5,600 \mathrm{~dB}$ & 1.12 & $\mathrm{~S}$ & $150,933 \mathrm{aA}$ & 30.18 & S & $28.46^{* *}$ \\
\hline CNPH 691 & $7,200 \mathrm{cC}$ & 1.44 & $\mathrm{~S}$ & $20,533 \mathrm{cB}$ & 4.10 & $\mathrm{~S}$ & $226,400 \mathrm{aA}$ & 45.28 & S & $16.69^{* *}$ \\
\hline CNPH 692 & $130,200 \mathrm{bA}$ & 26.04 & $\mathrm{~S}$ & $18,666 \mathrm{cB}$ & 3.73 & $\mathrm{~S}$ & $252,533 \mathrm{aA}$ & 50.50 & $\mathrm{~S}$ & $8.87^{* * *}$ \\
\hline CNPH 693 & $9,000 \mathrm{cB}$ & 1.80 & $S$ & $4,800 \mathrm{eC}$ & 0.96 & $\mathrm{R}$ & 239,466 aA & 47.89 & S & $30.63^{* *}$ \\
\hline BRS Mari & $319,500 \mathrm{aA}$ & 63.84 & $S$ & $3,466 \mathrm{eB}$ & 0.69 & $\mathrm{R}$ & $265,600 \mathrm{aA}$ & 53.12 & S & $49.07^{* *}$ \\
\hline BRS Moema & $365,100 \mathrm{aA}$ & 73.02 & S & $4,800 \mathrm{~dB}$ & 0.96 & $\mathrm{R}$ & $238,933 \mathrm{aA}$ & 47.78 & S & $29.27^{* *}$ \\
\hline 'St ${ }^{\mathrm{a}}$ Cruz Kada' & $823,800 \mathrm{aA}$ & 164.76 & $S$ & $586,133 \mathrm{aA}$ & 117.22 & $\mathrm{~S}$ & $754,400 \mathrm{aA}$ & 150.88 & S & $0.17^{\mathrm{ns}}$ \\
\hline Test F & $15.67^{* *}$ & & & $15,52^{* * *}$ & & & $0.59^{\mathrm{ns}}$ & & & \\
\hline
\end{tabular}

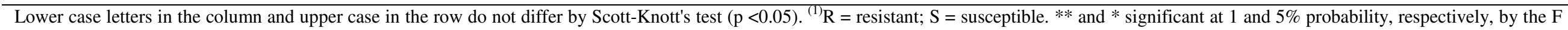
test. 
Table 7. Slicing of interactions between genotypes and root-knot nematodes species for number of eggs and second-stage juveniles per gram of root.

\begin{tabular}{|c|c|c|c|c|c|c|c|c|c|c|}
\hline \multirow{2}{*}{ Genotypes } & \multicolumn{3}{|c|}{ M. incognita raça 3} & \multicolumn{3}{|l|}{ M.javanica } & \multicolumn{3}{|l|}{ M. enterolobii } & \multirow[t]{2}{*}{ Test F } \\
\hline & NEJGR & RI & $\mathbf{R}^{(\mathbf{1})}$ & NEJGR & RI & $\mathbf{R}^{(\mathbf{1})}$ & NEJGR & RI & $\mathbf{R}^{(1)}$ & \\
\hline CNPH 64 & $1,123.36 \mathrm{~dB}$ & 4.29 & VR & $1,225.90 \mathrm{cB}$ & 11.09 & MoR & $12,396.68 \mathrm{aA}$ & 93.84 & $\mathrm{~S}$ & $25,43^{* *}$ \\
\hline CNPH 145 & $1,495.14 \mathrm{cB}$ & 5.72 & VR & $402.00 \mathrm{dC}$ & 3.63 & VR & $9,296.48 \mathrm{aA}$ & 70.38 & $\mathrm{~S}$ & $25,81^{* * *}$ \\
\hline CNPH 191 & $11,726.29 \mathrm{aA}$ & 44.86 & SR & $670.78 \mathrm{cB}$ & 6.06 & VR & $6,929.61 \mathrm{aA}$ & 52.46 & $\mathrm{~S}$ & $26,19^{* * *}$ \\
\hline CNPH 194 & $7,150.84 \mathrm{aA}$ & 27.35 & SR & $2,187.59 \mathrm{bB}$ & 19.79 & MoR & $10,478.40 \mathrm{aA}$ & 79.32 & S & $9,57^{* *}$ \\
\hline CNPH 198 & $8,348.06 \mathrm{aA}$ & 31.93 & SR & $6,727.79 \mathrm{aA}$ & 60.86 & S & $12,241.61 \mathrm{aA}$ & 92.67 & $\mathrm{~S}$ & $1,00^{\mathrm{ns}}$ \\
\hline CNPH 199 & $9,825.67 \mathrm{aA}$ & 37.59 & SR & $260.94 \mathrm{eB}$ & 2.35 & VR & $14,459.24 \mathrm{aA}$ & 109.46 & $\mathrm{~S}$ & $64,11^{* * *}$ \\
\hline CNPH 200 & $2,795.20 \mathrm{cB}$ & 10.69 & VR & $804.12 \mathrm{cC}$ & 7.27 & VR & $8,350.14 \mathrm{aA}$ & 63.21 & $\mathrm{~S}$ & $16,31^{* *}$ \\
\hline CNPH 291 & $14,548.66 \mathrm{aA}$ & 55.65 & S & $446.37 \mathrm{~dB}$ & 4.04 & VR & $13,153.47 \mathrm{aA}$ & 99.57 & $\mathrm{~S}$ & $51,49^{* *}$ \\
\hline CNPH 292 & $10,339.96 \mathrm{aA}$ & 39.55 & SR & $786.50 \mathrm{cB}$ & 7.11 & VR & $11,014.27 \mathrm{aA}$ & 83.38 & $\mathrm{~S}$ & $27,16^{* *}$ \\
\hline CNPH 295 & $9,682.42 \mathrm{aA}$ & 37.04 & SR & $623.68 \mathrm{cB}$ & 5.64 & VR & $10,511.07 \mathrm{aA}$ & 79.57 & $\mathrm{~S}$ & $28,23^{* *}$ \\
\hline CNPH 296 & $3,591.22 \mathrm{bB}$ & 13.73 & MoR & $1,993.44 \mathrm{bB}$ & 18.03 & MoR & $9,968.17 \mathrm{aA}$ & 75.46 & $\mathrm{~S}$ & $8,13^{* *}$ \\
\hline CNPH 297 & $7,019.69 \mathrm{aA}$ & 26.85 & SR & $453.67 \mathrm{~dB}$ & 4.10 & VR & $10,411.38 \mathrm{aA}$ & 78.82 & $S$ & $34,19^{* * *}$ \\
\hline CNPH 432 & $14,475.79 \mathrm{aA}$ & 55.38 & S & $46.80 \mathrm{gB}$ & 0.42 & $\mathrm{HR}$ & $8,410.04 \mathrm{aA}$ & 63.66 & $S$ & $149,04^{* *}$ \\
\hline CNPH 433 & $6,888.88 \mathrm{aA}$ & 26.35 & SR & $303.61 \mathrm{~dB}$ & 2.74 & VR & $6,707.06 \mathrm{aA}$ & 50.77 & $\mathrm{~S}$ & $37,26^{* * *}$ \\
\hline CNPH 580 & $9,058.01 \mathrm{aA}$ & 34.65 & SR & $273.14 \mathrm{eB}$ & 2.47 & VR & $7,373.27 \mathrm{aA}$ & 55.81 & $\mathrm{~S}$ & $44,76^{* *}$ \\
\hline CNPH 581 & $11,788.94 \mathrm{aA}$ & 45.10 & SR & $473.84 \mathrm{~dB}$ & 4.28 & VR & $6,901.10 \mathrm{aA}$ & 52.24 & $\mathrm{~S}$ & $32,93^{* * *}$ \\
\hline CNPH 582 & $9,422.74 \mathrm{aA}$ & 36.04 & SR & $133.08 \mathrm{fB}$ & 1.20 & VR & 7,698.67 aA & 58.28 & $\mathrm{~S}$ & $92,68^{* * *}$ \\
\hline CNPH 583 & $8,118.23 \mathrm{aA}$ & 31.05 & SR & $110.82 \mathrm{fB}$ & 1.00 & VR & $9,304.55 \mathrm{aA}$ & 70.44 & $\mathrm{~S}$ & $100,54^{* *}$ \\
\hline CNPH 593 & $7,490.50 \mathrm{aA}$ & 28.65 & SR & $248.53 \mathrm{eB}$ & 2.25 & VR & $6,704.43 \mathrm{aA}$ & 50.75 & $\mathrm{~S}$ & $47,29^{* * *}$ \\
\hline CNPH 602 & $9,499.52 \mathrm{aA}$ & 36.34 & SR & $433.03 \mathrm{~dB}$ & 3.91 & VR & $9,621.93 \mathrm{aA}$ & 72.84 & $\mathrm{~S}$ & $35,72^{* *}$ \\
\hline CNPH 640 & $11,180.69 \mathrm{aA}$ & 42.77 & SR & $132.50 \mathrm{fC}$ & 1.19 & VR & $4,125.80 \mathrm{aB}$ & 31.23 & SR & $87,06^{* * *}$ \\
\hline CNPH 641 & $7,015.02 \mathrm{aA}$ & 26.83 & SR & $549.58 \mathrm{~dB}$ & 4.97 & VR & $9,496.78 \mathrm{aA}$ & 71.89 & $S$ & $28,80^{* *}$ \\
\hline CNPH 642 & $16,652.44 \mathrm{aA}$ & 63.70 & $\mathrm{~S}$ & $281.29 \mathrm{eB}$ & 2.54 & VR & $8,836.46 \mathrm{aA}$ & 66.89 & $\mathrm{~S}$ & $59,83^{* * *}$ \\
\hline CNPH 644 & $11,939.05 \mathrm{aA}$ & 45.67 & SR & $4,157.01 \mathrm{bB}$ & 37.61 & SR & $11,579.66 \mathrm{aA}$ & 87.66 & $\mathrm{~S}$ & $5,96^{* *}$ \\
\hline CNPH 646 & $16,697.10 \mathrm{aA}$ & 63.87 & $\mathrm{~S}$ & $92.71 \mathrm{gB}$ & 0.83 & VR & $7,986.12 \mathrm{aA}$ & 60.46 & S & $134,56^{* *}$ \\
\hline CNPH 677 & $1,366.79 \mathrm{cB}$ & 5.23 & VR & $130.18 \mathrm{eC}$ & 1.17 & VR & $5,033.73 \mathrm{aA}$ & 38.10 & SR & $37,60^{* * *}$ \\
\hline CNPH 680 & $126.75 \mathrm{fB}$ & 0.48 & HR & $132.39 \mathrm{fB}$ & 1.19 & VR & $9,619.99 \mathrm{aA}$ & 72.82 & $\mathrm{~S}$ & $102,06^{* * *}$ \\
\hline CNPH 682 & $85.50 \mathrm{fC}$ & 0.32 & HR & $332.67 \mathrm{~dB}$ & 3.01 & VR & $9,442.30 \mathrm{aA}$ & 71.48 & $S$ & $76,97^{* *}$ \\
\hline CNPH 683 & $4,388.50 \mathrm{bA}$ & 16.79 & MoR & $123.07 \mathrm{fB}$ & 1.11 & VR & $8,644.44 \mathrm{aA}$ & 65.44 & S & $70,10^{* * *}$ \\
\hline CNPH 684 & $2,075.23 \mathrm{cB}$ & 7.94 & VR & $151.65 \mathrm{eC}$ & 1.37 & VR & $4,828.06 \mathrm{aA}$ & 36.55 & SR & $36,51^{* *}$ \\
\hline CNPH 687 & $10,176.10 \mathrm{aA}$ & 38.93 & SR & $371.33 \mathrm{~dB}$ & 3.35 & VR & $11,834.46 \mathrm{aA}$ & 89.59 & $\mathrm{~S}$ & $45,48^{* *}$ \\
\hline CNPH 688 & $4,511.66 \mathrm{bB}$ & 17.26 & MoR & $1,149.94 \mathrm{cC}$ & 10.40 & VR & $12,827.71 \mathrm{aA}$ & 97.11 & S & $16,53^{* *}$ \\
\hline CNPH 690 & $224.66 \mathrm{fB}$ & 0.86 & HR & $242.74 \mathrm{eB}$ & 2.19 & VR & $5,490.78 \mathrm{aA}$ & 41.56 & SR & $44,43^{* *}$ \\
\hline CNPH 691 & $231.12 \mathrm{eB}$ & 0.88 & HR & $494.11 \mathrm{~dB}$ & 4.47 & VR & $10,257.15 \mathrm{aA}$ & 77.65 & S & $45,37^{* *}$ \\
\hline CNPH 692 & $8,687.54 \mathrm{aA}$ & 33.23 & SR & $769.67 \mathrm{cB}$ & 6.96 & VR & $7,137.88 \mathrm{aA}$ & 54.03 & $\mathrm{~S}$ & $20,47^{* * *}$ \\
\hline CNPH 693 & $341.57 \mathrm{eB}$ & 1.30 & VR & $132.52 \mathrm{fC}$ & 1.19 & VR & $5,935.57 \mathrm{aA}$ & 44.93 & SR & $51,29^{* *}$ \\
\hline BRS Mari & $8,478.08 \mathrm{aA}$ & 32.43 & SR & $67.80 \mathrm{fB}$ & 0.61 & HR & $4,685.39 \mathrm{aA}$ & 35.47 & SR & $89,32^{* * *}$ \\
\hline BRS Moema & $9,543.73 \mathrm{aA}$ & 36.51 & SR & $130.77 \mathrm{eB}$ & 1.18 & VR & $10,773.94 \mathrm{aA}$ & 81.56 & $\mathrm{~S}$ & $69,75^{* * *}$ \\
\hline 'St ${ }^{\mathrm{a}}$ Cruz Kada' & $26,139.35 \mathrm{aA}$ & 100.00 & $\mathrm{~S}$ & $11,053.37 \mathrm{aA}$ & 100.00 & $\mathrm{~S}$ & $13,209.16 \mathrm{aA}$ & 100.00 & $\mathrm{~S}$ & $2,37^{\mathrm{ns}}$ \\
\hline Test $\mathrm{F}$ & $27.90^{* * *}$ & & & $22.76^{* *}$ & & & $1,11^{\mathrm{ns}}$ & & & \\
\hline
\end{tabular}

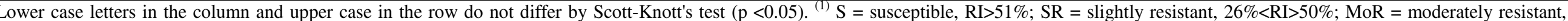

$11 \%<\mathrm{RI}>25 \%$; VR $=$ very resistant, $1 \%<\mathrm{RI}>10 \% ; \mathrm{HR}=$ highly resistant, $\mathrm{IR}<1 \%$. ** and $*$ significant at 1 and $5 \%$ probability, respectively, ${ }^{\mathrm{ns}} \mathrm{Not}$ significant, by the $\mathrm{F}$ test. 
With regard to $M$. enterolobii, with the exception of the $C$. frutescens line, it was observed a high reproduction in the genotypes evaluated in both experiments, obtaining high values of TNEJ and NEJGR. Despite the difference between the genotypes in the first experiment, by the Scott-Knott test, presenting two distinct groups, all genotypes were classified as susceptible by the Oostenbrink (1966) methodology with RF greater than 21.54. In experiment 2, all genotypes were classified in the same group, confirming susceptibility of all materials. Regarding Taylor (1967) classification method, in both experiments, the genotypes were grouped, by the Scott-Knott test, into a single group. However, there was divergence for the reaction, with seven genotypes slightly resistant and the others susceptible (Tables 4 and 7).

The high susceptibility of $C$. annuum to $M$. enterolobii is reported in several studies. Gonçalves et al. (2014) evaluated 13 accessions of $C$. annuum and observed RI from 36.90 to 397.70 , being characterized as slightly resistant to susceptible. Oliveira et al. (2009) tested different Capsicum species and verified that all accessions belonging to C. annuum were susceptible to $M$. enterolobii.

The low proportion of genotypes resistant to Meloidogyne spp. is stated in studies with Capsicum (MELO et al., 2011; PINHEIRO et al., 2013; GONÇALVES et al., 2014). Pinheiro et al. (2014) evaluated the resistance of 13 genotypes of Capsicum and verified that eight genotypes were susceptible to $M$. incognita and $M$. javanica, and for $M$. enterolobii, all genotypes were susceptible.

In general, classifications by index and reproduction factor were effective for the identification of genotypes resistant to $M$. incognita race 3, M. javanica and M. enterolobii (Tables 3, 4, 6 and 7). However, the classification proposed by Taylor (1967) provided a broader distribution of classes (I, HR, VR, MoR, SR and S), allowing more flexibility in classification, while the Oostenbrink (1966) methodology classified the genotypes exclusively as resistant (R) or susceptible (S). The classification of Oostenbrink (1966) becomes safer to select resistant genotypes, since it is based on the ratio of the initial and final numbers of nematode eggs and second-stage juveniles. In contrast, the classification of Taylor (1967) is based on the proportion of eggs and second-stage juveniles of nematodes, involving the highly susceptible control
(Andrade-Junior et al., 2016), and in this study it was used the 'Santa Cruz Kada' tomato, that is classified in different genus and species of the Capsicum species, although they belong to the same botanical family. Therefore, classification by the reproduction factor (OOSTENBRINK, 1966) is more suitable for selection of resistant genotypes.

As regards the multiple resistances to the root-knot nematodes species, only the genotypes $\mathrm{CNPH}$ 185, CNPH 187 and CNPH 680 were considered resistant to $M$. incognita race 3 and $M$. javanica, simultaneously. However, the genotypes described were not resistant to $M$. enterolobii.

Bitencourt and Silva (2010) points out the ability of $M$. enterolobii to reproduce in plants resistant to other species of Meloidogyne spp, such as the commercial hybrid Snooker, which has a pyramid of the $M e 1$ and $M e 3 / M e 7$ genes, responsible for resistance to $M$. incognita, $M$. arenaria and M. javanica (PINHEIRO et al., 2015).

In Brazil, to date, there are no reports of $C$. annuum genotypes with simultaneous resistance to $M$. incognita, $M$. javanica and $M$. enterolobii with potential to be used as rootstocks in the control of infested areas. The first study on resistance in Capsicum spp. to these nematodes was developed by Oliveira (2007), who observed that only one genotype of $C$. frutescens is resistant simultaneously to $M$. incognita and $M$. javanica, presented resistance to $M$. enterolobii. However, this genotype was the only one that showed incompatibility for grafting, as the plants that were grafted onto this genotype had the lowest height, productivity and fruit quality (OLIVEIRA et al., 2009). It is necessary the continuity of studies that are engaged in the search for genotypes with multiple resistances to root-knot nematodes, and that are good rootstocks candidates for cropping sweet pepper and/or to be used in breeding programs.

\section{CONCLUSIONS}

The genotypes CNPH 185, CNPH 187 and CNPH 680 are resistant to $M$. incognita race 3 and $M$. javanica, however, with no resistance to $M$. enterolobii.

The line of $C$. frutescens is the only genotype that shows multiple resistances to the three species of root-knot nematodes.

RESUMO: O presente trabalho teve por objetivo avaliar acessos de Capsicum quanto à resistência a Meloidogyne incognita raça 3, Meloidogyne javanica e Meloidogyne enterolobii. Foram realizados dois experimentos, com diferentes genótipos de pimentas e pimentões, em delineamento inteiramente casualizado sendo o primeiro em esquema 
fatorial 31 x 3 com 27 genótipos de Capsicum annuum, duas cultivares de pimenta, uma linhagem de Capsicum frutescens, o tomateiro 'Santa Cruz Kada' e três espécies de nematoides (M. incognita raça 3, M. javanica e M. enterolobii). No segundo experimento foi utilizado esquema fatorial 39 × 3 com 36 acessos de $C$. annuum, duas cultivares de pimenta, o tomateiro 'Santa Cruz Kada' e três espécies de nematoides mencionadas anteriormente. Avaliou-se o número total de ovos e juvenis de segundo estádio (NTOJ), número de ovos e juvenis de segundo estádio por grama de raízes (NOJGR), índice de reprodução (IR) e fator de reprodução (FR). Com base no FR e IR os genótipos CNPH 185, CNPH 187 e CNPH 680 foram resistentes e muito resistentes a $M$. incognita raça 3 e $M$. javanica, simultaneamente. A linhagem de $C$. frutescens apresentou resistência às três espécies de nematoides de galha.

PALAVRAS-CHAVE: Meloidogyne incognita. Meloidogyne javanica. Meloidogyne enterolobii. Pimentas e pimentões. Reação.

\section{REFERENCES}

ANDRADE JÚNIOR, V. C.; GOMES, J. A. A.; OLIVEIRA, C. M.; AZEVEDO, A. M.; FERNANDES, J. S. C.; GOMES, L. A. A.; MALUF, W. R. Resistência de clones de batatadoce a Meloidogyne javanica. Horticultura Brasileira, Brasília, v. 34, n. 1, p. 130-136, 2016. http://dx.doi.org/10.1590/S0102053620160000100020 .

BARbOSA, J. C.; MALDONADO JÚNIOR, W. Experimentação Agronômica \& AgroEstat: Sistema para análises estatísticas de ensaios agronômicos. Jaboticabal: UNESP, 2015. 396 p.

BITENCOURT, N. V.; SILVA, G. S. Reprodução de Meloidogyne enterolobii em Olerícolas. Nematologia Brasileira, Piracicaba, v. 34, n. 3, p. 181-183, 2010.

BRITO, J. A.; STANLEY, J. D.; KAUR, R.; CETINTAS, R.; DI VITO, M.; THIES, J. A.; DICKSON, D. W. Effects of the Mi1, N and Tabasco genes on infection and reproduction of Meloidogyne mayaguensis on tomato and pepper genotypes. Journal of Nematology, Florida, v. 39, n. 4, p. 327-332, 2007.

BÜTTOW, M. V.; BARBIERI, R. L.; NEITZKE, R. S.; HEIDEN. G.; CARVALHO, F. I. F. Diversidade genética entre acessos de pimentas e pimentões da Embrapa Clima Temperado. Ciência Rural, Santa Maria, v. 40, n. 6, p. 1264-1269, 2010. https://doi.org/10.1590/S0103-84782010000600004

DIAS-ARIEIRA, C. R.; CUNHA T. P. L.; CHIAMOLERA, F. M.; PUERARI, H. H.; BIELA, F.; SANTANA, S. M. Reaction of vegetables and aromatic plants to Meloidogyne javanica and M. incognita. Horticultura Brasileira, Brasília, v. 30, n. 2, p. 322-326, 2012. http://dx.doi.org/10.1590/S0102-05362012000200023

GONÇALVES, L. S. A.; GOMES, V. M.; ROBAINA, R. R.; VALIM, R. H.; RODRIGUES, R.; ARANHA, F. M. Resistance to root-knot nematode (Meloidogyne enterolobii) in Capsicum spp accessions. Revista Agrária, Recife, v. 9, n. 1, p. 49-52, 2014. http://dx.doi.org/ DOI:10.5039/agraria.v9i1a3496.

https://doi.org/10.5039/agraria.v9i1a3496

HUSSAIN, M. A.; MUKHTAR, T.; KAYANI, M. Z. Characterization of susceptibility and resistance responses to root-knot nematode (Meloidogyne incognita) infection in okra germplasm. Pakistan Journal of Agricultural Science, Faisalabad v. 51, n. 2, p. 309-314, 2014.

HUSSEY, R. S.; BARKER, K. R. A comparasion of methods of collecting inocula of Meloidogyne spp. including a new technique. Plant Disease Reporter, Washington, v. 57, p. 1025-1028, 1973.

IEA. INSTITUTO DE ECONOMIA AGRÍCOLA. Estatísticas de produção agropecuária paulista, 2016. Disponível em: < http://ciagri.iea.sp.gov.br/nia1/subjetiva.aspx?cod_sis=1\&idioma=1 >. Acesso em: $20 \mathrm{de}$ Maio de 2016. 
LIU, B.; REN, J.; ZHANG, Y.; AN, J.; CHEN, M.; CHEN, H.; XU, C.; REN, H. A new grafted rootstock against root-knot nematode for cucumber, melon, and watermelon. Agronomy for Sustainable Development, Paris, v. 35, n. 1, p. 251-259, 2015. https://doi.org/10.1007/s13593-014-0234-5

LÓPES-PÉREZ, J. A.; STRANGE, M.; KALOSHIAN, I.; PLOEG, A. T. Differencial response of Mi generesistant tomato rootstocks to root-knot nematodes (Meloidogyne incognita). Crop Protection, Guildford, v. 25, n. 4, p. 382-388, 2006. https://doi.org/10.1016/j.cropro.2005.07.001.

https://doi.org/10.1016/j.cropro.2005.07.001

MELO, O. D.; MALUF, W. R.; GONÇALVES, R. J. S.; NETO, A. C. G.; GOMES, L. A. A.; CARVALHO, R. C. Triagem de genótipos de hortaliças para resistência a Meloidogyne enterolobii. Pesquisa Agropecuária Brasileira. Brasília, v. 46, n. 8, p. 829-835, 2011. http://dx.doi.org/10.1590/S0100-204X2011000800007.

MOURA, M. F. ; MARUBAYASHI, J. M. ; MITUTI, T.; GIORIA, R. ; KOBORI, R. F. ; PAVAN, M. A. ; KRAUSE-SAKATE, R. Análise comparativa da região codificadora para a proteína capsidial de isolados de PepYMV e PVY coletados em pimentão. Summa Phytopathologica, Botucatu, v. 38, n. 1, p. 93-96, 2012. http://dx.doi.org/10.1590/S0100-54052012000100017

OLIVEIRA, D. C. Enxertia de plantas de pimentão em Capsicum spp. no manejo de nematóides de galha. 2007. 134p. Tese (Doutorado). Universidade Estadual Paulista - Faculdade de Ciências Agrárias e Veterinárias, Jaboticabal.

OLIVEIRA, C. D.; BRAZ, L. T.; SANTOS, J. M.; BANZATTO, D. A.; OLIVEIRA, P. R. Resistência de pimentas a nematóides de galha e compatibilidade enxerto/porta-enxerto entre híbridos de pimentão e pimentas. Horticultura Brasileira, Brasília, v. 27, n. 4, p. 520-526, 2009. http://dx.doi.org/10.1590/S010205362009000400019.

OOSTENBRINK, M. Major characteristics of the relation between nematodes and plants. Mededelingen Landbouw, v. 66, p. 1-46, 1966.

MOENS, M.; PERRY, N. R.; STARR, F. L. Meloidogyne species - a diverse group of novel and important plant parasites. In: PERRY, R. N.; MOENS, M.; STARR, J. L. Root-knot nematodes. Wallingford: CAB International. 2009. p. 1-17. https://doi.org/10.1079/9781845934927.0001

PIMENTA, S.; MENEZES, D.; NEDER, D. G.; MELO, R. A.; ARAÚJO, A. L. R.; MARANHÃO, E. A. A. Adaptability and stability of pepper hybrids under conventional and organic production systems. Horticultura Brasileira, Brasília, v. 34, n. 2, p. 168-174, 2016. http://dx.doi.org/10.1590/S0102-053620160000200004.

PINHEIRO, J. B.; REIFSCHNEIDER, F. J. B.; PEREIRA, R. B.; MOITA, A. W. Reprodução de Meloidogyne spp. em Capsicum spp. Nematologia brasileira, Piracicaba, v. 37, p. 20-25, 2013.

PINHEIRO, J. B.; REIFSCHNEIDER, F. J. B.; PEREIRA, R. B.; MOITA, A. W. Reação de genótipos de Capsicum ao nematoide-das-galha. Horticultura Brasileira, Brasília, v. 32, n. 2, p. 371-375, 2014. http://dx.doi.org/10.1590/S0102-05362014000300022.

PINHEIRO, J. B.; BOITEUX, L. S.; ALMEIDA, M. R. A.; PEREIRA, R. B.; GALHARDO, L. C. S.; CARNEIRO, R. M. D. G. First Report of Meloidogyne enterolobii in Capsicum Rootstocks Carrying The Me 1 and $M e 3 / M e 7$ Genes in Central Brazil. Nematropica, Florida, v. 45, n. 2, p. 184-188, 2015.

TAYLOR, A. L. Introduction to research on plant nematology: an FAO guide to the study and control of the plant parasitic nematodes. Rome: Food and Agricultural Organization of the United Nations, 1967. 133p. 